\title{
Is There Any Correlation Between MRI Features of Breast Lesions of BI-RADs Category 4 with Histopathologic Results?
}

\author{
Ghazaleh Soofi, Amirhossein Sarrami, and Maryam Farghadani \\ "Corresponding author: Ghazaleh Soofi. E-mail: ghazalehsoofi@gmail.com \\ Received 2016 December 21; Accepted 2017 February 08.
}

\begin{abstract}
Objectives: To evaluate the correlation of MRI features of breast lesions of BI-RADs category 4 with histopathologic results.

Methods: In a prospective study between December 2013 and April 2015, patients with suspicious mammographic and/or ultrasound findings referred for Breast MRI were evaluated. All the breast MRI were interpreted by a trained radiologist. Patients with lesions of BI-RADs category 4 were enrolled with written informed consent. In each patient mass lesion (ML) or non-mass lesion (NML) were determined. In patients with ML, shape (irregular or microlobulated), border (ill-defined or spiculated), contrast media distribution (inhomogenous or ring enhancement), initial and post initial contrast enhancement (strong enhancement, rapid, plateau, or continuous washout) were assessed. In patients with NML, pattern of enhancement (focal, linear, ductal, segmental or heterogeneous regional) was assessed. A Follow up program were taken with mean 3 to 12 months. The patients which underwent core needle biopsy or open biopsy were summoned.

Results: 81 females aged 24 to 67 years (mean $43 \pm 9.2$ ) met the inclusion criteria and had adequate samples for histopathologic study. 28 (34\%) patients had ML, 46 (56.8\%) patients had NML and 7 (8.6\%) had both ML and NML. Rate of normal result, epithelial hyperplasia, fibrocystic changes, fibroadenoma, papilloma, atypical hyperplasia, mixed fibrocystic changes and atypical hyperplasia, ductal carcinoma in situ, and invasive carcinoma were $2.5 \%, 12.3 \%, 19.8 \%, 8.6 \%, 3.7 \%, 19.8 \%, 4.9 \%, 14.8 \%$, and $13.6 \%$, respectively. In statistical analysis none of the MRI features has significant correlation with any specific histopathologic diagnosis.

Conclusions: This study showed that a wide spectrum of histopathologic results are seen in BI-RADs category 4. However, in this sample volume none of the MRI features in this BIRADs category has significant correlation with any specific histopathologic diagnosis.
\end{abstract}

This is an abstract presented in the 33rd Iranian congress of radiology (ICR) and the 15th congress of Iranian radiographic science association (IRSA). 